\title{
COVID-19 Pandemic- A Curse to the Physical Well-Being of Every Individual in Lock-Down
}

\author{
Pratik Arun Phansopkar ${ }^{1}$, Waqar Mohsin Naqvi², Arti Isherkumar Sahu ${ }^{3}$ \\ ${ }^{1}$ Department of Musculoskeletal Physiotherapy, Ravi Nair Physiotherapy College, Datta Meghe Institute of \\ Medical Sciences, Wardha, Maharashtra, India. ${ }^{2}$ Department of Community Physiotherapy, Ravi Nair \\ Physiotherapy College, Datta Meghe Institute of Medical Sciences, Wardha, Maharashtra, India. ${ }^{3}$ Department \\ of Community Physiotherapy, Ravi Nair Physiotherapy College, Datta Meghe Institute of Medical Sciences, \\ Wardha, Maharashtra, India.
}

\section{ABSTRACT}

\section{BACKGROUND}

The 2019 novel coronavirus (COVID-19) has rapidly spread from its origin in Wuhan City of China to other countries. In response to the rapid spread of COVID19, preventive measures such as social distancing have resulted in the global closure of all means of entertainment or recreational activities. Owing to the lockdown phase and more tendencies for sedentary lifestyle than earlier, there is a higher probability of the population to suffer from musculoskeletal issues during this phase. This can further result in various physical health issues in terms of reduced endurance, reduced muscle strength, obesity, and metabolic problems associated with decreased physical activity (PA), etc. Aerobic exercise has shown that the peripheral number of lymphocytes in the blood is significantly raised; precipitating the idea that exercise helps to "stimulate" the immune system during exercise. PA has various health benefits including reduced incidence of disease and decreased systemic inflammation. A physically active lifestyle has a direct impact on an individual's state of well-being, quality of life, and cognitive function. Thus, PA is related to a decreased chance of cognitive decline and dementia. Previous studies have shown that daily PA leads to lower death rates and infection incidence rates. Both physical activity and exercise are positively related to beneficial metabolic and immunological health outcomes. Besides, they have proven to be effective with direct effects on physical health for most of the chronic diseases. Routine exercises improve the improve physical wellbeing. Therefore, individual prescription and guidance are required to establish an effective exercise program aimed in sustaining or enhancing the key health-related components of physical fitness. This article gives an overview of the musculoskeletal awareness in lockdown phase owing to increased sedentary lifestyle during the lockdown phase.

\section{KEY WORDS}

COVID-19, Quarantine, Lockdown, Sedentary Lifestyle, Exercise, Physical Activity
Corresponding Author: Dr. Pratik Arun Phansopkar, Assistant Professor, Department of Musculoskeletal Physiotherapy, Ravi Nair Physiotherapy College, Datta Meghe Institute of Medical Sciences, Wardha, Maharashtra, India. E-mail: drpratik77@gmail.com

DOI: $10.14260 / \mathrm{jemds} / 2020 / 556$

How to Cite This Article: Phansopkar PA,Naqvi WM, Sahu AI.COVID19 pandemic: a curse to the physical wellbeing of every individual in lock-down. J Evolution Med Dent Sci 2020;9(35):25612566, DOI: 10.14260/jemds/2020/556

Submission 28-05-2020,

Peer Review 22-07-2020,

Acceptance 28-07-2020,

Published 31-08-2020.

Copyright (C) 2020 JEMDS. This is an open access article distributed under Creative Commons Attribution License [Attribution 4.0 International (CC BY 4.0)] 
Coronavirus disease (COVID-19) caused by a virus referred to as SARS- CoV-2 has landed up to be a global pandemic affecting people worldwide. ${ }^{1}$ Coronaviruses are a huge family of viruses which mainly cause illness such as respiratory disease, including symptoms like nasal congestion, dry cough, fever, running nose, tiredness, which is commonly quoted as COVID-19. The strain of this virus first originated from Wuhan city of China and this new strain of coronavirus, later began to spread in other countries with an increasing number of cases. ${ }^{2,3}$ During this tough time of the pandemic, WHO recommended all countries to encourage preparedness activities to fight against the spread of illness. Few necessary precautionary measures like maintaining respiratory hygiene, market practices like social distancing, were also made compulsory for all to prevent the nosocomial spread of the virus. ${ }^{4}$ To date, children and adults have been moderately affected with fewer deaths. No specific coronavirus anti-viral or vaccine with proven results has yet been discovered.

Due to a rapid rise in the cases of COVID-19, the governments worldwide have declared a strict lockdown until the disease is under control. Implementing strict lockdown and social distancing measures alleviated the quick spread of the virus, thus were effective in reducing the mortality rate and prevention of disease transmission. ${ }^{5}$ The lockdown phase led to a drastic change in people's routine, lifestyle and work pattern. As people were encouraged to work from home and use Tele-media, their physical health was predominantly compromised. One of the major causes of the decline in physical wellbeing could be due to the decreased in physical activity (PA), where physical activity accounted for routine activities performed by an individual at home, based on the job, or for conveyance reasons. ${ }^{6}$ The decline in physical activity led to a sedentary lifestyle due to the extended behavioural periods resulting in low energy ( e.g. sitting and lying) expenditure. ${ }^{6}$ Sedentary behaviour(SB) can further result in various physical health issues in terms of reduced endurance, reduced muscle strength, obesity, metabolic problems associated with the decreased PA, etc. Exercises are recommended to overcome physical inactivity and sedentary behaviour, which are programmed, organized and repetitive to enhance or maintain physical wellbeing. ${ }^{6}$ The lockdown phase and more tendencies for the sedentary lifestyle and lack of outdoor activity have resulted in a higher probability for the population to serve a price for musculoskeletal issues during this phase of lockdown. This article gives an overview of the impact of the sedentary lifestyle physical wellbeing during the lockdown phase. The main aim of this article is to understand the idea and the concept of physical well-being and awareness during this time of lockdown wherein we all are experiencing a change in the pattern of our lifestyle by staying indoors.

\section{Global Trends of Sedentary Behaviour during Lockdown:}

The world seems to have adapted sedentary behaviour(SB) as another pandemic amid the COVID-19 pandemic. ${ }^{7}$ Although SB was declared a pandemic in 2012, and prime associations acknowledged this crisis and championed attempts to enhance PA, yet there are still insufficient trends in PA. Various studies indicate that SB will continue even after COVID-19 pandemic subsides, and the health and economic effects of the SB pandemic will continue to be severe. SB has also been demonstrated to be an important forecaster of unfavourable health outcomes. COVID-19 precautionary measures leading to lockdown have accelerated the SB pandemic. ${ }^{7}$ Due to lockdown measures, several prospects to remain physically active have been discontinued, which include school-based physical education and sports events, wellness centers and metropolitan parks. ${ }^{7}$ Besides, several fitness centers have provided free online exercise workouts to help people stay healthy at home. It is difficult to disregard the correlation between the health risks associated with COVID-19 and the current SB status. ${ }^{7}$

Due to the increased usage of handheld devices such as mobile phones, tablets, game controls, personal digital assistants and portable media players, musculoskeletal problems are at a peak during the lockdown period. ${ }^{8}$ The studies have reported that the prolonged use of these devices induces constant mechanical stress on the musculoskeletal system, psychological strain, ergonomic risks leading to musculoskeletal disorders, commonly neck pain..$^{9,10}$ As this lockdown period has employed a lot of people with work from home schedules, there has been a usual increase in texting activity, video conferencing, hours of internet activity like online courses, webinars and all other important works are now being done by relying on internet and technology. If not paid attention to these, it can further lead to problems like postural impairment, text next syndrome, nerve compression, muscle fatigue, spasm etc. which may cause pain, discomfort and other musculoskeletal symptoms.

\section{The Interesting Connection between Physical Health and Immunological Health:}

Studies have shown that a physically active regime diminishes the risk of developing infections. ${ }^{6}$ It is generally observed during intense aerobic exercise that the peripheral number of lymphocytes in the blood is significantly heightened, giving rise to the idea that PA and exercise help to "stimulate" the immune system during exercise. This is due to elevated blood pressure and shear forces during exercise. Furthermore, the prime controller is the adrenergic stimulation of beta-2-adrenergic receptors on the lymphocyte surface due to the release of adrenaline during exercise. This leads to endothelial detachment and consequent lymphocyte recirculation into the blood-stream. ${ }^{6}$ These immune cells supposedly recognize and kill cells, which are pathogen-infected or have become weakened or malignant, according to the theory of acute stress/immune enhancement exercise. ${ }^{6}$ Evidence from animal studies supports evidence linking regular levels of PA to improved immune skills in people and suggests that the immunological advantages of PA can be especially beneficial in enhancing otherwise weak responses in older age. ${ }^{6}$ Besides, preliminary evidence shows that PA and routine exercise can also restrict or postpone the weakening of the immune system. Therefore, exercise must be promoted, especially for those who are at the highest risk of infection and who may acquire the maximum exercise-induced immune competency benefits. Throughout moderate to intense aerobic exercise, the tissue macrophage anti-pathogenic activity occurs in tandem with the increased immunoglobulin recirculation, neutrophils, cytotoxic $\mathrm{T}$ cells, anti-inflammatory cytokines, natural killer cells, too immature B cells, all of 
which play vital roles in immune defense function and metabolic safety. ${ }^{11}$ Injury and inflammation of the exercise-induced muscle tissue evoke a resilient innate immune response, which involves monocytes, granulocytes and macrophages. Immune-specific proteins are generated to modulate the innate immune response, involving oxylipins to initiate, mediate, and resolve this process.(11) The thorough, exercise-induced perturbation in metabolites, proteins and lipid mediators has a direct effect on immune function, decreasing immune cells' ability to raise oxygen consumption levels after activation. ${ }^{11}$ Activation of the immune system is correlated to oxygen and biosynthetic demands, and metabolic reprogramming by the immune cells is required to produce adequate energy to accomplish these demands. ${ }^{11}$ PA facilitates improved yet intermittent immunesurveillance, and daily PA offers several health benefits comprising reduced incidence of disease and decreased systemic inflammation. ${ }^{11}$ Several epidemiological studies show that daily PA correlates with lower death rates and infection incidence rates. ${ }^{11}$ Besides, there is evidence of enhanced antibody responses to influenza immunization in individuals involved in regular exercise training schemes. ${ }^{11}$ Principally, exercise facilitates the recirculation of prime immune cells, and by various pathways, intervenes an anti-inflammatory and antioxidant states. Evidence show that older adults are more susceptible to infections and exhibit lower immune responses to vaccines compared to their younger counterparts. $^{12}$ Studies have shown that the reduced inflammatory biomarkers or memory to the naïve $T$ lymphocyte ratio can contribute to the benefit of chronic moderate aerobic exercise on immune responses, even though it was not tested in their studies.

\section{The Odd Relationship between Sedentary Behaviour Obesity, and Infection:}

As regards lean subjects, SB or reduced PA is typical of obese patients. Reduced PA, in itself or facilitated by insulin resistance, impairs the immune response to infections at many immune response stages comprising macrophage activation and pro-inflammatory cytokine inhibition. ${ }^{13}$ Obesity and obese diabetics have a change in the inborn and adaptive immune response at various stages, depicted by persistent and low-grade inflammation. ${ }^{13}$ Upon presentation of an antigen, the chronic inflammation associated with obesity causes decreased macrophage activation, which further leads to dampened pro-inflammatory cytokine production.(13) This results in low vaccination efficacy of obese subjects and this explains the appearance in the obese population of antiviral-resistant and vaccine-free variants. ${ }^{13}$ In obese and obese diabetic patients, $\mathrm{B}$ and $\mathrm{T}$ cell responses are also blunted, resulting in increased susceptibility and delay in viral infection resolution. Obese patients may demonstrate an altered number and function of lymphocytes resulting in altered memory $\mathrm{T}$ cell responses and vaccine efficacy. ${ }^{13}$ Obesity stops all virus-specific CD8 $+\mathrm{T}$ cell responses and seasonal influenza vaccine antibody responses, resulting in suboptimal macrophage activity and maturation. ${ }^{13}$ A robust response from $\mathrm{CD} 8+\mathrm{T}$ cells plays a significant role in defending against new strains and subtypes of influenza viruses. Since the incubation time for COVID-19 is aforethought 14 days and obese subjects exhibit prolonged shedding of the virus, quarantine must be extended in obese compared to lean subjects. ${ }^{13}$ Both PA and exercise are positively related to beneficial metabolic and immunological health outcomes. ${ }^{13} \mathrm{PA}$ has an immunemodulatory action, which focuses on enhancing weight loss and immune modulation. Both mortality and morbidity can be dampened with routine exercise, although weight loss might not be significant. Various studies indicate that routine PA and exercises increase the levels of TLR (toll-like receptor)-mediated cytokine production during microbial infection, enhancing host resistance to pathogen invasion. ${ }^{13}$ Furthermore, exercise enhances the antioxidant defense system, as well as reducing oxidative stress. Regular PA emerges as a cornerstone, as a precautionary measure to improve the host defense against influenza viral infection and other metabolic diseases in obese subjects. ${ }^{13}$

\section{Role of Physical Activity (PA) and Exercises in Musculoskeletal, Neuromotor and Cardiorespiratory Fitness:}

For seemingly healthy adults of all ages, a thorough exercise program should involve resistance, cardiorespiratory, neuromotor and endurance exercise of quality and adequate quantity, as explained in this outline. Decreasing overall duration spent in sedentary activities and interlacing brief physical activity bouts and standing in sedentary activity cycles should be a priority in favour of every person, regardless of their exercise behaviour. PA carried out in this manner improves mental and physical wellbeing including fitness in most people.(14)Furthermore, an exercise regime which does not involve all exercise modules or achieves less than the recommended exercise magnitudes (frequency, intensity and duration) is likely to be advantageous, particularly in adapted inactive people. ${ }^{14}$ Due to the significant individual variations in the reaction to an exercise regime, the exercise recommendation is best tailored depending upon the individual responses.Exercising is impactful only if an individual is involved in it. Consequently, reflecting on a person's desires and pleasure, and integrating behavioural improvement and health behaviour, theory approaches into an exercise routine will improve daily exercise acceptance and short-term maintenance. Efficient approaches for minimizing CVD and musculoskeletal exercise risks include screening and informing on early signs and symptoms of CVD during exercise in individuals, consultation with a professional to monitor health, and adherence to a variety of exercise prescription elements including warm-up, cool-down, an incremental progression of exercise intensity and volume, and correct training techniques. ${ }^{14}$ The guidance of a qualified and skilful fitness professional will improve commitment to work out, and potentially decrease the likelihood of adverse events with CHD in the high risk individuals. ${ }^{15,16}$ For the Adults, particularly beginner exercisers and individuals with disabilities or health issues, it is possible to experience the gains of PA and exercise regime under the observation of a skilled fitness professional. 


\section{Benefits of Physical Activity and Exercise on Metabolic Disease RiskFactors, Cardiovascular and Cardiorespiratory Fitness:}

Long-term sedentary behaviours (SB) are directly linked with aggravated risk of CHD mortality and depression, unfavourable events with CHD, low lipoprotein lipase activity, increased waist circumference, blood pressure and aggravated biomarkers of long standing disease such as lipoproteins, blood glucose, and insulin. ${ }^{15,16}$ SB is harmful even among people who fulfil current recommendations for daily physical activity. If sedentary behaviours are interrupted by short periods of PA or standing, these adverse physiological effects can be attenuated. ${ }^{16} \mathrm{~A}$ lifestyle that is physically active promotes state of well-being, feelings of "strength', better quality of life, and increased cognitive functions, and is linked with a low risk of dementia and cognitive impairment.PA enhances lipoprotein profile, reduces blood pressure, biomarkers of CHD, C-reactive protein, enhances insulin sensitivity, and plays a very important role in weight control. Each component of physical fitness (i.e., muscle strength, muscular fitness (endurance), cardiorespiratory fitness, flexibility, and fitness for the neuromotor and body composition) is likely to influence some aspects of health. Increased levels of cardiorespiratory and muscular fitness are each linked with low risks to poor health. The associations among biological risk factors, clinical health outcomes, and cardiorespiratory fitness seem to be alike to those for physical activity: generally stable older adults and middle-aged with higher baseline cardiorespiratory fitness, and those who increase fitness in time have a low risk of all the causes and CVD morbidity and mortality. ${ }^{17}$ The dosage of physical activity needed to obtain a particular health advantage can be further explained by associating specific quantities of physical activity with appropriate levels of cardio-respiratory fitness to promote health benefits.

Either moderate or vigorous-intensity workout or together may be initiated to fulfil existing guidelines for the workout, provided that the requirement for overall energy consumption is met. There is also a higher amount of exercise in the vigorous intensity state in almost all studies where the benefit is observed for vigorous exercise compared with moderate intensity exercise. The intensity of exercise is a vital parameter of the physiological responses to the preparation. As per the training principle of overload, exercising lower than a minimum threshold or intensity will not sufficiently test the body to improve physiological parameters and increase VO2 Max. ${ }^{18}$ Many studies provide evidence for an average level of intensity for the benefits, but lack reliable results which seem to be linked to the initial stage of health and conditioning of the individuals. A threshold of intensity of exercise can vary based on the level of fitness, and an exact threshold can be difficult to determine precisely to enhance cardiorespiratory fitness. Cardiorespiratory exercise decreases several risk factors for cardio metabolic diseases, although the magnitude of the effect is adequate, differs according to the exercise program characteristics and the individual, and it appears that a change in any of the cardio metabolic risk factor occurs regardless of a change in another factor. ${ }^{19}$ the Higher muscle strength levels are linked with substantially improved cardio metabolic risk factor statuses, fewer CVD accidents, the lower risk of all-cause mortality, nonfatal disease and the lower risk of functional impairment. In individuals with prehypertension or stage 1 hypertension, there are enhancements in the composition of the body, blood sugar levels, insulin sensitivity and blood pressure apart from greater strength. Importantly, an exercise that aids muscle strength and weight often beneficially improves bone mass volumes (bone mineral content and density) and bone strength of the stressed individual bones and can cater as a vital measure to avoid delay or reverse the bone mass loss in individuals with osteoporosis.14,20,21 As a risk factor for osteoarthritis growth, muscle weakness has been recognized, weight training may lower the possibility of musculoskeletal derangements from developing. In persons with osteoarthritis resistance,the training may reduce disability and pain.

The Musculoskeletal fitness can be successfully attained through a resistance training program which consists of endurance, strength, and power functional variables. Incorporating weights such as dumbbells, equipment with stacked weights or pneumatic resistance and even TheraBand resistance bands, they enhance muscle fitness.Flexibility exercises can improve balance and postural stability, especially if done in combination with resistance exercises. ${ }^{22,23}$ Improvements in range of movement can be attained by numerous flexibility exercises, such as static stretching, ballistic methods or "bouncing" stretches, Proprioceptive neuromuscular facilitation (PNF) methods and dynamic or slow movement stretching. Functional fitness training or neuromotor exercise training combines motor skills such as coordination, agility, gait, balance, and proprioceptive training. Neuromotor exercise program is beneficial in the aspect of a comprehensive exercise regime for the elderly, in particular, to enhance muscle strength, mobility, balance and minimize the risk of falling. ${ }^{14}$ Balance training and agility can remarkably reduce the fear of falling and risk of falling, and therefore prevent unexpected falls, although more suggestive and decisive evidence is required to support this related finding. ${ }^{24} \mathrm{~A}$ particular training regime's magnitude of the effect can differ drastically among people in middle and elderly aged groups, and some individuals may not respond as expected. Variations in training results among individuals are correlated with various variables comprising the features of the training program, environmental considerations, and various individual aspects such as genetic variation, fitness level, and daily physical activity, physiological and psychological and social influences. Metabolic health measures, muscle strength, and functional efficiency can be sustained at minimum of at least one session per week of exercise from mild to moderate intensity. ${ }^{14}$

\section{The Necessity and Benefits of Physical Activity during Lockdown: \\ Studies have shown that only normal, moderate and intense aerobic exercise has improved the immune response to influenza vaccination related to SB. ${ }^{12}$ There is sufficient evidence to show that long-term moderate aerobic exercise facilitates in reducing the risk of infections and enhancing the immune response to vaccination and pneumonia. ${ }^{12}$ Lack of physical activity and our pattern of using handheld devices}


influence the normal body mechanics of a body. Improvising the basic physical activity routine will help to maintain the health of the overall musculoskeletal system and functional performance. So a routine of physical activity is important to keep the body balanced and fit, to maintain overall circulation and to maintain one's strength and endurance. Exercises enhance various parameters by supplying nutrients to the musculoskeletal system, alleviating joint pain and stiffness, enhancing flexibility and mobility, building muscle strength, increasing or preserving bone density, lowering stress rates, releasing endorphins and enkephalins (to alleviate anxiety) and improving overall body oxygenation, thereby enhancing the feeling of well-being.

Maintaining an active lifestyle during lockdown is very vital for the overall population's wellbeing. ${ }^{25} \mathrm{PA}$ and exercise have evidence to be a successful treatment with direct effects on physical wellbeing for most of the chronic diseases. Exercise has been deemed to be the true polyp ill based on epidemiological proof of its therapeutic or preventive effects and taking into account the key biological intermediary involved. 25 Special consideration should be given to the elderly due to the impact of PA and exercise in older people on many diseases as well as additional impacts on the characteristics of ageing and associated diseases. ${ }^{25}$ Therefore, it is very important for the health of the general population not to interrupt or change people's lifestyle during lockdown phase, and to sustain an active lifestyle at homes. ${ }^{25}$

\section{The Role of Physical Activity inCombating Sedentary Behaviour:}

Each one of us desires for a pain-free body. Maintaining physical health has proven effective in preventing many health consequences with direct impacts on both physical and mental health. Despite the fact that outdoor activities are usually more accessible, varied, and have added facilities and resources to do some kind of physical exercise, there are yet plenty of opportunities to practice at home during a quarantine. ${ }^{25}$ Briefly, the rationale for encouraging PA to enhance physical health is that cardiorespiratory activity and muscle strength influence the physiological functions of the body. For this, exercise and physical activity are necessary to combat the physical and mental consequences and seriousness of Covid-19 during the quarantine. 7,25 Guidance and a specific prescription are required to ensure an effective exercise regimen aimed at sustaining or enhancing the key health-related elements of physical fitness. A mixture of many physiological factors underpins the maintenance of muscular strength and proper posture. ${ }^{26}$

A multi-component exercise program is considered the most appropriate for people rather than following a single discipline. It involves activities related to aerobics, resistance, balance, coordination, and mobility training. Several experts have also recently proposed integrating the idea of cognitive stimulation during the exercise training session. ${ }^{25}$ According to world health organization (WHO), a minimum of 150 minutes exercise duration per week, that is, $30 \mathrm{~min} /$ day for 5 days a week, (however, these can be divided in 10-10-10 minute bouts) is recommended.? Along with this, a routine of basic indoor physical activity is advisable to keep the body free from any musculoskeletal problems associated with a sedentary lifestyle. Indoor activities like yoga, breathing exercises, changing of position after every 15-30 minutes, stretching your body, ankle pumps, brisk walking in some open area in the house, will help to maintain good circulation, physical health, a posture, which will ensure physical well-being. Exercise duration of 150 to 300 minutes over 5-7 days per week should be done regularly with adaptation in volume and intensity. Besides, resistance exercises of at least 2-3 days a week may be recommended. Exercise at a moderate intensity is well recognized to strengthen the immune system. ${ }^{25}$ If one does not have specific exercise materials, the following activities can be easily carried out at home to reduce SB:

1. Resistance training, such as stepping up and down, mini squats holding a chair, or carrying weight items (water, rice etc.).

2. Aerobic exercises, such as walking or dancing in the hall.

3. Exercise in balance, such as walking on the toes or heels or overcoming obstacles, walking on a line on the floor. ${ }^{25}$

To be well prepared is the most important aspect of fighting the future pandemic. ${ }^{27}$ In crucial phase like this, it is vital to be informed and important for all the individuals to unfold the accurate and right facts for their advancement in the society. ${ }^{28}$

\section{CONCLUSIONS}

The COVID-19 breakdown has led to the lockdown all over the world, impacting people's daily life. Sedentary behaviour and reduced physical activity have resulted in altered immune response and increased risk of obesity among people. Sedentary lifestyle is associated with altered physical wellbeing in people of all age group, thus making it a concern needing immediate attention. SB and reduced PA can be mitigated by performing regular exercises and correcting posture, wherever required. Thus, keeping a check on sedentary behavior in the lockdown phase is of great importance to optimize, maintain \& improve the overall performance of the body.

Financial or Other Competing Interests: None.

\section{REFERENCES}

[1] Berhe B, Legese H, Degefa H, et al. Global epidemiology, pathogenesis, immune response, diagnosis, treatment, economic and psychological impact, challenges, and future prevention of COVID-19: a scoping review.medRxiv: Public and Global Health 2020. http://medrxiv.org/lookup/doi/10.1101/2020.04.02.20 051052

[2] Sardu C, Gambardella J, Morelli MB, et al. Hypertension, thrombosis, kidney failure, and diabetes: is COVID-19 an endothelial disease? A comprehensive evaluation of clinical and basic evidence. J Clin Med 2020;9(5):1417. 
[3] Singhal T. A review of coronavirus disease-2019 (COVID19). Indian J Pediatr 2020;87(4):281-6.

[4] Arias-Palencia NM, Solera-Martínez M, Gracia-Marco L, et al. Levels and patterns of objectively assessed physical activity and compliance with different public health guidelines in university students PLoS One 2015;10(11):e0141977.

[5] Gustafsson E, Johnson PW, Hagberg M. Thumb postures and physical loads during mobile phone use -a comparison of young adults with and without musculoskeletal symptoms. J Electromyogr Kinesiol 2010;20(1):127-35.

[6] Campbell JP, Turner JE. Debunking the myth of exerciseinduced immune suppression: redefining the impact of exercise on immunological health across the lifespan. Front Immunol 2018;9:648.

[7] Hall G, Laddu DR, Phillips SA, et al. A tale of two pandemics: how will COVID-19 and global trends in physical inactivity and sedentary behaviour affect one another? Prog Cardiovasc Dis 2020;500330620(20)30077-3.

[8] Sharan D, Mohandoss M, Ranganathan R, et al. Musculoskeletal disorders of the upper extremities due to extensive usage of hand held devices. Ann Occup Environ Med 2014;26(1):22.

[9] Lee M, Hong Y, Lee S, et al. The effects of smartphone use on upper extremity muscle activity and pain threshold. J Phys Ther Sci 2015;27(6):1743-5.

[10] Berolo S, Wells RP, Amick BC. Musculoskeletal symptoms among mobile hand-held device users and their relationship to device use: a preliminary study in a Canadian university population. Appl Ergon 2011;42(2):371-8

[11] Nieman DC, Wentz LM. The compelling link between physical activity and the body's defense system. J Sport Health Sci 2019;8(3):201-17.

[12] Song Y, Ren F, Sun D, et al. Benefits of exercise on influenza or pneumonia in older adults: a systematic review. Int J Environ Res Public Health 2020;17(8):2655.

[13] Luzi L, Radaelli MG. Influenza and obesity: its odd relationship and the lessons for COVID-19 pandemic. Acta Diabetol 2020;57(6):759-64.

[14] Garber CE, Blissmer B, Deschenes MR, et al. American College of Sports Medicine position stand. Quantity and quality of exercise for developing and maintaining cardiorespiratory, musculoskeletal, and neuromotor fitness in apparently healthy adults: guidance for prescribing exercise. Med Sci Sports Exerc 2011;43(7):1334-59.

[15] Teychenne M, Ball K, Salmon J. Sedentary behaviour and depression among adults: a review. Int J Behav Med 2010;17(4):246-54.
[16] Owen N, Healy GN, Matthews CE, et al. Too much sitting: the population health science of sedentary behaviour. Exerc Sport Sci Rev 2010;38(3):105-13.

[17] Lee DC, Sui X, Ortega FB, et al. Comparisons of leisuretime physical activity and cardiorespiratory fitness as predictors of all-cause mortality in men and women. Br J Sports Med 2011;45(6):504-10.

[18] Thompson PD, Arena R, Riebe D, et al. ACSM's new preparticipation health screening recommendations from ACSM'sguidelines for exercise testing and prescription, ninth edition. Curr Sports Med Rep 2013;12(4):215-7.

[19] Oeland AM, Laessoe $U$, Olesen AV, et al. Impact of exercise on patients with depression and anxiety. Nord J Psychiatry 2010;64(3):210-7.

[20] Collier SR, Kanaley JA, Carhart R, et al. Cardiac autonomic function and baroreflex changes following 4 weeks of resistance versus aerobic training in individuals with pre-hypertension. Acta Physiol (Oxf) 2009;195(3):33948.

[21] Hunter GR, Brock DW, Byrne NM, et al. Exercise training prevents regain of visceral fat for 1 year following weight loss. Obesity (Silver Spring) 2010;18(4):690-5.

[22] Costa PB, Graves BS, Whitehurst M, et al. The acute effects of different durations of static stretching on dynamic balance performance. J Strength Cond Res 2009;23(1):141-7.

[23] Bird M, Hill KD, Ball M, et al. The long-term benefits of a multi-component exercise intervention to balance and mobility in healthy older adults. Arch Gerontol Geriatr 2011;52(2):211-6.

[24] Price JPK, Arthur N, Macstephen AO. Correlation between body mass index and peak expiratory flow rate of an indigenous nigerian population in the Niger delta region. Res J Recent Sci 2013;2(2):28-32.

[25] Jiménez-Pavón D, Carbonell-Baeza A, Lavie CJ. Physical exercise as therapy to fight against the mental and physical consequences of COVID-19 quarantine: special focus in older people. Prog Cardiovasc Dis2020;S00330620(20)30063-3.

[26] Suchomel TJ, Nimphius S, Bellon CR, et al. The importance of muscular strength: training considerations. Sports Med 2018;48(4):765-85.

[27] Naqvi W, Shah P. Fighting and chasing the rogue visrusCOVID 19. Int J Research in Pharmaceutical Sciences 2020;11(Spl 1):77-80.

[28] Dutta S, Acharya S, Shukla S, et al. COVID-19 pandemicrevisiting the myths. Int J Med Sci 2020;7(5):7-10. 